OPEN ACCESS

Edited by:

Lihua Xiao,

South China Agricultural

University, China

Reviewed by:

Meng Qi,

Tarim University, China

Hamed Mirjalali,

Shahid Beheshti University of Medical

Sciences, Iran

Soledad Fenoy Rodríguez, CEU San Pablo University, Spain

*Correspondence:

Żaneta Zajączkowska

zaneta.kopacz@umed.wroc.pl

Specialty section:

This article was submitted to Infectious Diseases - Surveillance,

Prevention and Treatment,

a section of the journal

Frontiers in Medicine

Received: 15 July 2021 Accepted: 03 September 2021

Published: 30 September 2021

Citation

Zajączkowska Ż, Akutko K, Kváč M, Sak B, Szydłowicz M, Hendrich $A B$, Iwańczak B and Kicia M (2021) Enterocytozoon Bieneusi Infects Children With Inflammatory Bowel

Disease Undergoing Immunosuppressive Treatment.

Front. Med. 8:741751. doi: 10.3389/fmed.2021.741751

\section{Enterocytozoon Bieneusi Infects Children With Inflammatory Bowel Disease Undergoing Immunosuppressive Treatment}

\author{
Żaneta Zajączkowska ${ }^{1 *}$, Katarzyna Akutko ${ }^{2}$, Martin Kváč ${ }^{3,4}$, Bohumil Sak ${ }^{3}$, \\ Magdalena Szydłowicz ${ }^{1}$, Andrzej B. Hendrich ${ }^{1}$, Barbara Iwańczak ${ }^{2}$ and Marta Kicia ${ }^{1}$ \\ ${ }^{1}$ Department of Biology and Medical Parasitology, Wrocław Medical University, Wrocław, Poland, ${ }^{2}$ Department and Clinic of \\ Paediatrics, Gastroenterology and Nutrition, Wrocław Medical University, Wrocław, Poland, ${ }^{3}$ Biology Centre of the Czech \\ Academy of Sciences, Institute of Parasitology, Ceské Budějovice, Czechia, ${ }^{4}$ Faculty of Agriculture, University of South \\ Bohemia, Ceské Budějovice, Czechia
}

Objectives: Patients with inflammatory bowel disease (IBD) are susceptible to intestinal opportunistic infections due to both defective mucosal immunity and altered immune response resulting from immunosuppressive treatment. Microsporidia infecting the gastrointestinal tract and causing diarrhoea can potentially affect the course of IBD.

Methods: Stool samples (90 IBD children and 121 healthy age-matched controls) were screened for Encephalitozoon spp. and Enterocytozoon bieneusi by microscopy and polymerase chain reaction followed by sequencing.

Results: E. bieneusi genotype D was found in seven out of 90 (7.8\%) IBD children. No children from the control group were infected, making the pathogen prevalence in the IBD group significant $(P=0.002)$. Furthermore, infection was confirmed only in patients receiving immunosuppressive treatment $(P=0.013)$.

Conclusions: Children with IBD are at risk of intestinal E. bieneusi infection, especially when receiving immunosuppressive treatment. Therefore, microsporidia should be considered as a significant infectious agent in this group of patients.

Keywords: Enterocytozoon bieneusi, inflammatory bowel disease, children, immunosuppressive treatment, molecular characterization

\section{INTRODUCTION}

Inflammatory bowel disease (IBD) is a complex, chronic inflammatory condition of the gastrointestinal tract developing as a result of the interaction of genetic and environmental factors. The two main types of IBD are Crohn's disease (CD) and ulcerative colitis (UC). Although the aetiology of IBD is still poorly understood, the role of the gut microbiota combined with genetic susceptibility in disease pathogenesis is highlighted (1). Since immunomodulators are commonly used in the treatment of IBD, patients' immune response is compromised making them more susceptible to opportunistic infections (2). Currently, IBD has evolved into a worldwide disorder with rising prevalence in paediatric populations and age is an additional, independent risk factor for opportunistic infections (3). To date, the majority of reported infections among IBD patients have been caused by bacteria and viruses (4). Reports on fungal infections in the course of IBD are comparatively rare $(5,6)$. 
Enterocytozoon bieneusi and species from the genus Encephalitozoon (E. intestinalis, E. cuniculi and E. hellem) are the most clinically significant microsporidia - opportunistic fungi infecting eukaryotic cells (7). Due to a wide range of animal hosts, microsporidial spores are distributed in the environment, contaminating water and food, and infecting hosts through faecal-oral or inhalation routes $(8,9)$. Since their spores initially develop in the epithelium of the small intestine, diarrhoea, abdominal pain, fever, nausea, malabsorption and weight loss are the most common symptoms of microsporidiosis (10). As opportunistic pathogen, microsporidia cause more severe and even fatal infection mainly in immunosuppressed patients, such as HIV-infected or organ and bone marrow transplant recipients $(11,12)$. In turn, in immunocompetent people such an infection usually manifests as a self-limiting diarrhoea or stays asymptomatic. Such a latent infection may, however, affect the course and symptoms of basic disease entity in patients with chronic disorders (13).

Here, we present the prevalence of microsporidia in the gastrointestinal tract of children with IBD, both with CD and UC. The role of microsporidial infection in the course of IBD has not been well-studied so far and available literature is restricted to adult patients only. To the best of our knowledge, this is the first report concerning microsporidial infection among IBD children.

\section{MATERIALS AND METHODS}

\section{Patients}

Samples from two groups of HIV-negative patients under the age of 18 years were analysed between 2014 and 2017: (i) children with IBD (study group; $n=90$ ), including children with CD (57/90) and UC (33/90), who had been under the care of the 2nd Department and Clinic of Paediatrics, Gastroenterology and Nutrition (Wroclaw Medical University, Poland) and (ii) healthy children (control group; $n=121$ ) matched by age with the study group. The revised Porto Criteria recommended by the European Society for Paediatric Gastroenterology, Hepatology and Nutrition (ESPGHAN) were used for the diagnosis of IBD (14). Clinical CD and UC activity were assessed according to the Paediatric Crohn's Disease Activity Index (PCDAI) and the Paediatric Ulcerative Colitis Activity Index (PUCAI), respectively. IBD patients were divided according to three clinical frameworks: (i) $43(47.8 \%)$ patients with active disease presenting at, or shortly after, diagnosis with no previous treatment for IBD, (ii) $20(22.2 \%)$ patients with remission (PCDAI/PUCAI scores $<10$ for at least 12 months) and (iii) 27 (30\%) patients with active disease (mild: PCDAI/PUCAI scores 11-25; moderate: PCDAI/PUCAI scores 26-50; severe: PCDAI/PUCAI scores $>51$ and symptoms of disease).

The immunosuppressive treatment was administered in $52.2 \%$ (47/90) of all IBD children, according to current clinical practise. Four groups of immunosuppressive drugs: (i) glucocorticoids, (ii) azathioprine, (iii) methotrexate and (iv) infliximab or adalimumab were implemented in various configurations as shown in Table 1. Of 47 patients with immunomodulatory treatment, 38 were treated with one drug only (80.9\%), eight with two drugs (17\%) and one with three drugs (2.1\%). Moreover,
TABLE 1 | Comparison of Enterocytozoon bieneusi-positive and -negative IBD children's basic characteristics.

\begin{tabular}{|c|c|c|c|}
\hline \multirow[t]{2}{*}{ Characteristic } & \multicolumn{2}{|c|}{ Enterocytozoon bieneusi } & \multirow[t]{2}{*}{$P$-value } \\
\hline & Positive $(N=7)$ & Negative $(N=83)$ & \\
\hline \multicolumn{4}{|l|}{ Age } \\
\hline Median years (range) & $11(10-17)$ & $14.5(6-17)$ & 0.117 \\
\hline \multicolumn{4}{|l|}{ Sex } \\
\hline Male & $3(42.9)$ & $48(57.8)$ & 0.461 \\
\hline Female & $4(57.1)$ & $35(42.2)$ & 0.461 \\
\hline \multicolumn{4}{|l|}{ Type of IBD } \\
\hline $\mathrm{CD}$ & $5(71.4)$ & $52(62.7)$ & 1.000 \\
\hline UC & $2(22.2)$ & $31(38.3)$ & 1.000 \\
\hline \multicolumn{4}{|l|}{ Symptoms } \\
\hline Fever & 0 & $2(2.4)$ & 1.000 \\
\hline Diarrhoea & $2(28.6)$ & $28(33.7)$ & 1.000 \\
\hline \multicolumn{4}{|l|}{ Microbiological diagnosis } \\
\hline Yersinia & 0 & $1(2.4)$ & 1.000 \\
\hline Salmonella group D & 0 & $1(2.4)$ & 1.000 \\
\hline Clostridium difficile toxins & 0 & $3(7.1)$ & 1.000 \\
\hline Metronidazole & $2(28.6)$ & $13(15.7)$ & 0.330 \\
\hline \multicolumn{4}{|l|}{ Immunosuppressive regimen } \\
\hline general immunosuppression & $7(100)$ & $40(48.2)$ & $0.013^{*}$ \\
\hline $\begin{array}{l}\text { glucocorticoids } \\
\text { monotherapy }\end{array}$ & 0 & $5(6.0)$ & 1.000 \\
\hline azathioprine monotherapy & $6(85.7)$ & $22(26.5)$ & $0.003^{*}$ \\
\hline $\begin{array}{l}\text { adalimumab/infliximab } \\
\text { monotherapy }\end{array}$ & 0 & $5(6.0)$ & 1.000 \\
\hline $\begin{array}{l}\text { azathioprine + } \\
\text { glucocorticoids }\end{array}$ & $1(14.3)$ & $7(8.4)$ & 0.491 \\
\hline $\begin{array}{l}\text { glucocorticoids }+ \\
\text { methotrexate }+ \text { infliximab }\end{array}$ & 0 & $1(1.2)$ & 1.000 \\
\hline
\end{tabular}

Data represent number (\%) unless otherwise indicated; $C D$, Crohn's disease; UC, ulcerative colitis; " $P$-value $<0.05$.

at the time of the study, $16.7 \%$ (15/90) of IBD children were being treated with metronidazole. Adequate antibiotics were administered in case of five children diagnosed with bacterial infection.

Apart from the diagnosis of IBD, there were no other criteria for participation in the study group. Among clinical data, diarrhoea (defined as at least 3 watery bowel movements per day) and fever (body temperature above $38^{\circ} \mathrm{C}$ ) at the time of examination were taken into account. Additionally, standard laboratory microbiological examinations were carried out among IBD children to identify infections with bacteria of the genus Salmonella, Shigella, Campylobacter, Yersinia and toxins $\mathrm{A}$ and $\mathrm{B}$ of Clostridium difficile during acute exacerbation of the basic disease. The following exclusion criteria have been applied for control group: (i) acute infections, (ii) inflammatory, autoimmune or immunodeficiency diseases, and (iii) no previous immunosuppressive treatment history of note.

\section{Sample Collection}

Single stool samples were collected into disposable sterile containers from all of the patients. In the case of three 
TABLE 2 | Sequences of oligonucleotides used for specific Enterocytozoon bieneusi and Encephalitozoon spp. rDNA amplification and direct sequencing.

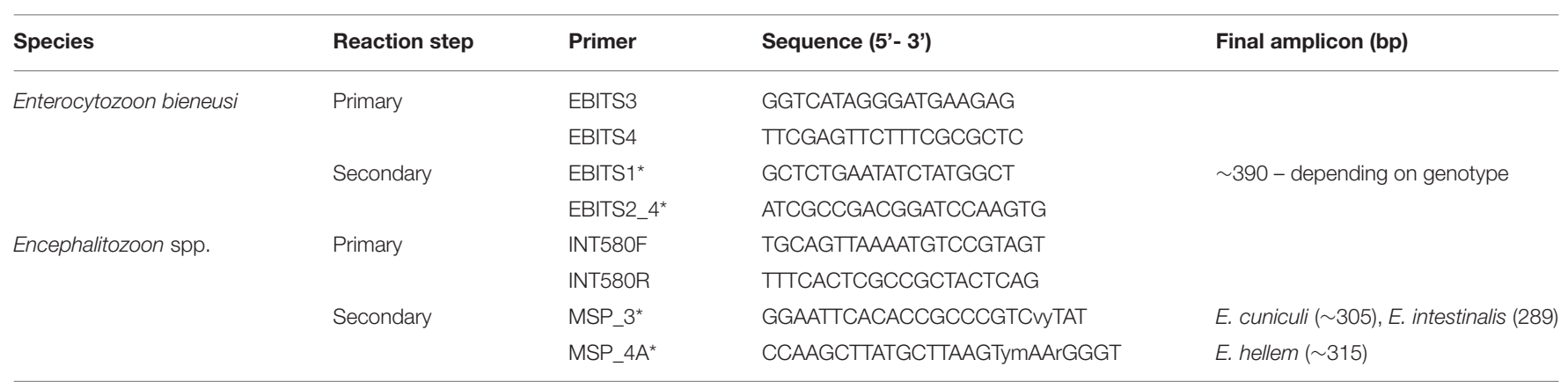

*oligonucleotides used for direct sequencing.

IBD children additional sampling after implementation of immunosuppressive treatment was performed. Each sample was split into two parts, one part was immediately fixed in high quality methanol for microscopy and second part was stored, without preservatives at $-20^{\circ} \mathrm{C}$ for $1-2$ weeks before molecular analyses.

\section{Microscopic Examination}

Standard Calcofluor M2R staining method was used to detect microsporidial spores (15). Briefly, dry methanol-fixed smears were stained with $0.1 \%$ Calcofluor M2R (Sigma-Aldrich, St. Louis, MO, USA) in phosphate-buffered saline (PBS) for $10 \mathrm{~min}$, rinsed gently with PBS, stained with $0.5 \%$ Evans blue (SigmaAldrich, St. Louis, MO, USA) in distilled water for $30 \mathrm{~s}$, rinsed again in PBS, and allowed to air dry. Stained slides were examined by fluorescence microscopy using UV light and at a magnification of $1,000 x$.

\section{DNA Isolation}

For DNA extraction, a total of $200 \mathrm{mg}$ of stool was initially homogenised by bead disruption using a Precellys 24 Instrument (Bertin Technologies, France). Genomic DNA (gDNA) was extracted using the GeneMATRIX Stool DNA Purification Kit (EurX, Gdańsk, Poland) according to the manufacturers' instructions and stored at $-20^{\circ} \mathrm{C}$ for molecular analyses.

\section{Molecular Examination}

gDNA was analysed by genus-specific nested polymerase chain reaction (nested-PCR) protocols amplifying a partial sequence of a small subunit of ribosomal RNA gene (16S rRNA), the entire ITS (internal transcribed spacer) region, and a partial sequence of the 5.8S rRNA gene of E. bieneusi and Encephalitozoon spp (1618 ). Sequences of primers used in primary and secondary nestedPCR are shown in Table 2. Negative (molecular grade water) and positive controls (DNA extracted from E. bieneusi genotype CZ3, E. cuniculi genotype III spores) were included in each PCR amplification. All manipulations during DNA extractions and amplifications were performed in separate areas of the laboratory, in laminar flow cabinets, previously decontaminated by UV light and disinfectants with international aseptic attestations. Disposable pipettes, tubes and reagent aliquots were used to avoid contamination.

\section{Sequencing and Phylogenetic Analyses}

Secondary PCR products were sequenced in both directions using the Sanger sequencing method. Amplification and sequencing of each sample were repeated twice. Nucleotide sequences were edited using the program ChromasPro 2.1.5 and aligned with each other and with reference sequences from GenBank (www.ncbi.nlm.nih.gov/blast) using MAFFT version 7 (http://mafft.cbrc.jp/alignment/software/). Phylogenetic analyses were performed using MEGA7 software and trees were inferred by the maximum likelihood (ML) method. Bootstrap support for branching was based on 1,000 pseudoreplicates.

\section{Statistical Analysis}

The Fisher's exact test was used to compare categorical variables (sex, type and activity of IBD, presence of fever and/or diarrhoea, applied immunosuppressive treatment and metronidazole) between microsporidia-positive and -negative patients, while the continuous variable (age) was compared using Student's $t$-test. A value of $P<0.05$ was considered significant.

\section{Ethics Approval Statement}

The study was approved by the Human Research Ethics Committee of Wrocław Medical University (KB-24/2014). Written consent was provided by the parents or legal guardians on behalf of all the paediatric patients involved in the study.

\section{RESULTS}

Among all IBD children $(n=90)$ the median age of patients was 14 years, and ranged between six and 17 years, while in children of the control group $(n=121)$ the median age was 10 years, and ranged between three and 16 years. Overall, the male-to-female ratio was $51(56.7 \%)$ to $39(43.3 \%)$ in the study, and $58(47.9 \%)$ to $63(52.1 \%)$ in the control group.

At the time of examination, diarrhoea and fever were observed in 30 and two IBD patients, respectively. Yersinia and Salmonella group D infections were confirmed in one child each, while Clostridium difficile toxins were identified in three children among 42 subjected to microbiological examination.

None of the children tested were positive for the presence of specific DNA of Encephalitozoon spp. Out of 90 IBD children, seven $(7.8 \%)$ were PCR positive for E. bieneusi (Table 1). 


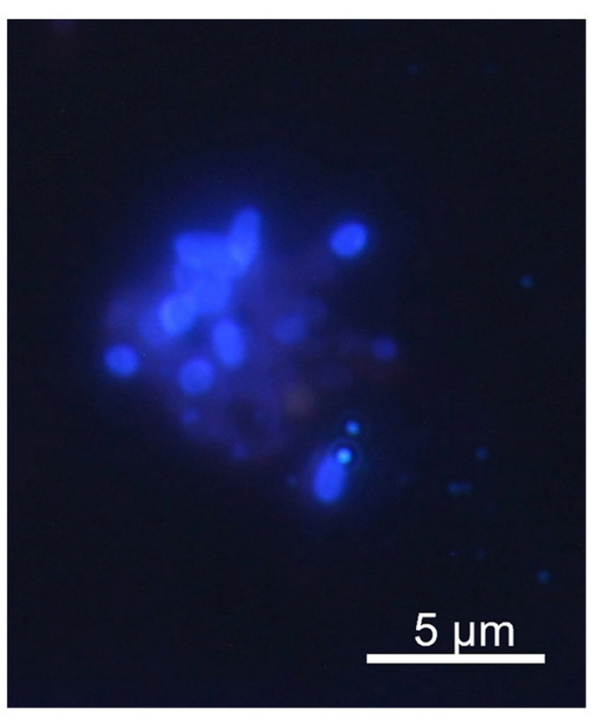

FIGURE 1 | Microsporidial spores identified in Calcofluor M2R staining of IBD patient stool.

No infection caused by these microsporidia was confirmed among children from the control group, making the E. bieneusi prevalence in IBD patients statistically significant $(P=0.002$; data not shown). Phylogenetic analysis revealed the presence of E. bieneusi genotype D in all samples. Spores were confirmed microscopically in five out of seven (71.4\%) samples (Figure 1). No co-infection with other screened bacteria was observed in any E. bieneusi-positive patient.

The E. bieneusi infection was confirmed exclusively in a group of children receiving immunosuppressive treatment, which is $14.9 \%(7 / 47)$ of the patients in that group $(P=0.013)$, regardless of its duration (1.5-43 months). Six out of seven (85.7\%) E. bieneusi-positive IBD patients received azathioprine as a single drug $(P=0.003)$, while one patient received simultaneous therapy with azathioprine and glucocorticoids. Out of three patients diagnosed before and after azathioprine implementation, two were positive for E. bieneusi, and infection was confirmed only after immunosuppressant treatment. E. bieneusi infection was observed in patients with various activity of IBD (Table 3 ). No significant associations were found for the other tested variables $(P>0.05$; Table 1$)$.

Detailed clinical and demographic characteristics of E. bieneusi-positive patients are shown in Table 3.

\section{DISCUSSION}

Routine examination of infectious agents among IBD individuals includes mainly bacteria and viruses. In our study we found E. bieneusi-intracellular parasitic fungus-in $7.8 \%$ of children with IBD, while none of the patients from the control group was infected, and this difference was statistically significant. Similar results have been shown in stool samples collected from adults with IBD receiving immunosuppressive agents, where $12.7 \%$ of patients were infected with E. bieneusi (19).

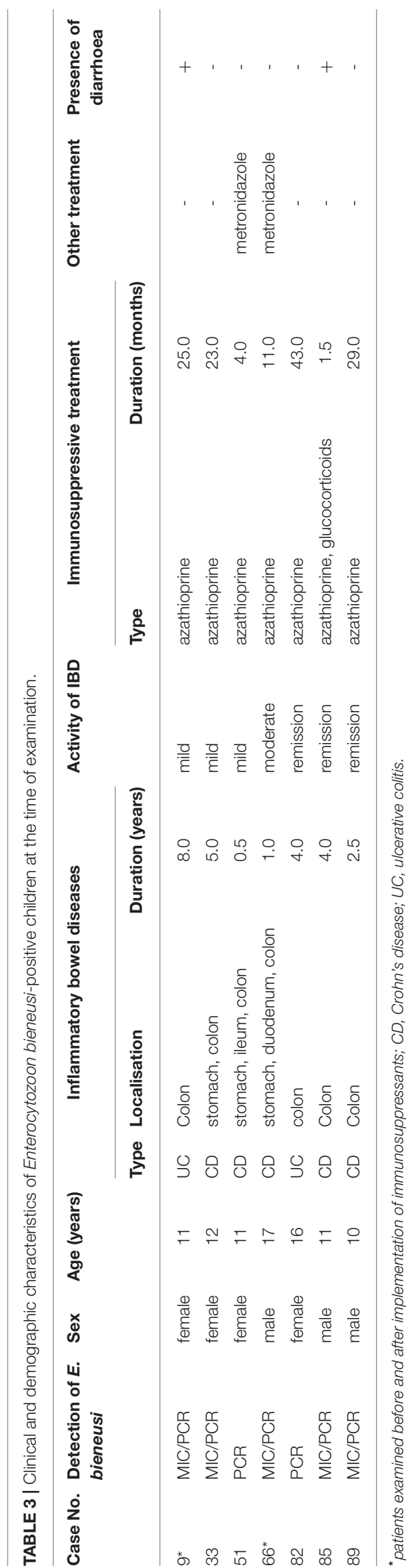


One of the reasons for increased susceptibility of IBD patients to such opportunistic infections and/or the reactivation of latent infection might be the immune deterioration as a result of immunosuppressive treatment implemented to achieve IBD remission and/or to prevent disease flares. Indeed, in our study E. bieneusi was confirmed exclusively in children with IBD receiving immunosuppressive treatment. Since the duration of immunosuppressive therapy in E. bieneusi-positive patients varied from 1.5 to 43 months, even temporary immunomodulatory treatment may be a risk factor for $E$. bieneusi infections. This may be confirmed by the detection of E. bieneusi infection in two of our patients after, but not before treatment with immunosuppressive drug, azathioprine.

On the other hand, the ongoing inflammatory process in the course of IBD might facilitate inhabitation of the intestine by pathogens. Since IBD affects the gastrointestinal tract, the influence of this disorder on increased susceptibility to infection may result from the degradation of the intestine and defective mucosal immunity (5). In the course of $\mathrm{CD}$ as well as of UC inflammatory infiltrates appear within the intestinal mucosa, destroying mucosal and, in case of UC, also submucosal membrane. Moreover, mucosal inflammation as a result of alterations in the structure and/or quantity of mucins and claudins, could make the intestine more vulnerable to penetration of pathogen $(20,21)$. It would allow the pathogen to persist in the intestinal mucosa, making the process chronic (5). Furthermore, microsporidia could persist and replicate inside macrophages and in this form, they can be transported throughout the host (22). Brdíčková et al. (23) have shown that other microsporidial species, E. cuniculi genotype II, migrates to inflammatory foci probably with the contribution of immune cells participating in its formation (23). If this is the case in patients suffering from chronic inflammatory process, subsequent worsening of the IBD clinical picture might occur.

Epidemiological studies have shown that microsporidiosis among individuals with an impaired immune system is symptomatic, including primarily diarrhoea and fever (12). Since diarrhoea might be a symptom of IBD as well, the difficulties in differentiation the causative agent in cases of infection is generally highlighted (24). Nevertheless, only a minority of microsporidia-positive samples were diarrheal and no significant association between infection and diarrhoea or fever was observed in patients in this study. However, as microsporidia can persist as a latent infection (25), there is a risk of development of symptoms, for instance as a result of increasing the immunosuppressive treatment dose. Moreover, infection with intracellular pathogens in patients with chronic inflammatory process may cause deepening in tissue damage in the place where inflammation persists and worsening of the clinical picture of the disease. Therefore, the need for diagnosing of such infections, especially in immunosuppressed patients, even asymptomatic, is emphasised by the risk of development of more severe symptoms, their incomprehensible impact on the course of the disease and the complexity or the effectiveness of the therapy process.

Interestingly, all of our patients were infected with E. bieneusi, similarly as in the report of Hasani et al. (19). Genotype D which is widespread among different groups of patients, both immunosuppressed an immunocompetent (12) and has a broad geographic range (26), was identified in all these samples. However, regardless of the high E. bieneusi prevalence among immunocompetent people (25), intestinal microsporidial infections, especially in immunosuppressed patients, can be associated also with Encephalitozoon species, mainly $E$. intestinalis and E. cuniculi. There are studies suggesting that metronidazole, drug used in IBD therapy, might be able to reduce the number of cells infected with Encephalitozoon species in vitro (27) and E. bieneusi responds only temporarily to treatment with metronidazole (28). However, according to the study by Lallo et al. (29) metronidazole is not effective against microsporidia. A similar conclusion can be drawn from clinical studies (30). Therefore, it is difficult to conclude if metronidazole treatment applied in $16.7 \%$ of IBD children had an influence on the microsporidial species identified in this study. Moreover, the question as to whether or not processes taking place in the intestine of IBD patients promote in some way inhabitation selectively by $E$. bieneusi remains unknown and requires detailed investigation. Nevertheless, detailed characterisation of microsporidial species is essential due to the fact that microsporidia treatment is limited and genus-specific. Albendazole is the drug of choice for Encephalitozoon spp. treatment and even though it is relatively well-tolerated, this drug has limited efficacy against $E$. bieneusi, especially in patients with impaired immune systems (30). Fumagillin reveals higher efficacy against $E$. bieneusi but shows toxicity and may not be fully effective in eradicating the pathogen (31). In such cases, rehabilitation of the immune system by dose reduction or temporary withdrawal of the immunosuppressive drug may be beneficial and lead to the pathogen elimination without the need for specific treatment (32).

In conclusion we have shown that children with IBD should be considered as a group at risk of microsporidial intestinal infections, especially when receiving immunomodulatory treatment. Even though we have not found any correlation between $E$. bieneusi infection and course of illness, the impact of such intestinal infection on the inflammatory processes in the bowel of IBD patients cannot be excluded. Since most of $E$. bieneusi-infected patients were asymptomatic such an unnoticed infection might develop to a chronic condition, as a result leading to progressive intestine devastation. Therefore, it seems that microsporidia should be considered during diagnosis of infectious agents affecting children suffering from IBD.

\section{DATA AVAILABILITY STATEMENT}

The raw data supporting the conclusions of this article will be made available by the authors, without undue reservation.

\section{ETHICS STATEMENT}

The study was approved by the Human Research Ethics Committee of Wrocław Medical University (KB-24/2014). 
Written informed consent to participate in this study was provided by the participants' legal guardian/next of kin.

\section{AUTHOR CONTRIBUTIONS}

ŻZ and MKi designed research. ŻZ, KA, MK, BS, MS, and MKi performed research and analysed data. $\dot{Z} Z, K A$, and MKi wrote the manuscript and MK, BS, MS, AH, and BI revised of the manuscript for important intellectual content. All authors had full access to all the data in the study and had final responsibility for the decision to submit the manuscript for publication.

\section{REFERENCES}

1. Khan I, Ullah N, Zha L, Bai Y, Khan A, Zhao T, et al. Alteration of gut microbiota in inflammatory bowel disease (IBD): cause or consequence? IBD treatment targeting the gut microbiome. Pathogens. (2019) 8:128. doi: 10.3390/pathogens8030126

2. Seyedian SS, Nokhostin F, Malamir MD. A review of the diagnosis, prevention, and treatment methods of inflammatory bowel disease. J Med Life. (2019) 12:113-22. doi: 10.25122/jml-2018-0075

3. Rosen MJ, Dhawan A, Saeed SA. Inflammatory bowel disease in children and adolescents. JAMA Pediatr. (2015) 169:105360. doi: 10.1001/jamapediatrics.2015.1982

4. Rahier JF, Magro F, Abreu C, Armuzzi A, Ben-Horin S, Chowers Y, et al. Second European evidence-based consensus on the prevention, diagnosis and management of opportunistic infections in inflammatory bowel disease. J Crohn Colitis. (2014) 8:443-68. doi: 10.1016/j.crohns.2013. 12.013

5. Andreu-Ballester JC, Garcia-Ballesteros C, Amigo V, Ballester F, Gil-Borrás R, Catalán-Serra I, et al. Microsporidia and its relation to Crohn's disease. A retrospective study. PLoS ONE. (2013) 8:e62107. doi: 10.1371/journal.pone.0062107

6. Beheshti-Maal A, Shahrokh S, Ansari S, Mirsamadi ES, Yadegar A, Mirjalali $\mathrm{H}$, et al. Gut mycobiome: the probable determinative role of fungi in IBD patients. Mycoses. (2021) 64:468-76. doi: 10.1111/myc. 13238

7. Gill EE, Fast NM. Assessing the microsporidia-fungi relationship: combined phylogenetic analysis of eight genes. Gene. (2006) 21:103-9. doi: 10.1016/j.gene.2006.02.023

8. Didier ES. Microsporidiosis: an emerging and opportunistic infection in humans and animals. Acta Trop. (2005) 94:6176. doi: 10.1016/j.actatropica.2005.01.010

9. Javanmard E, Mirjalali H, Niyyati M, Jalilzadeh E, Tabaei SJS, Aghdaei HA. Molecular and phylogenetic evidences of dispersion of humaninfecting microsporidia to vegetable farms via irrigation with treated wastewater: one-year follow up. Int J Hyg Environ Health. (2018) 221:64251. doi: 10.1016/j.ijheh.2018.03.007

10. Wasson K, Peper RL. Mammalian microsporidiosis. Vet Pathol. (2000) 37:113-28. doi: 10.1354/vp.37-2-113

11. Didier ES, Weiss LM. Microsporidiosis: current status. Curr Opin Infect Dis. (2006) 19:785-92. doi: 10.1097/01.qco.0000244055.46 382.23

12. Kicia M, Wesolowska M, Kopacz Z, Jakuszko K, Sak B, Květonová $\mathrm{D}$, et al. Prevalence and molecular characteristics of urinary and intestinal microsporidia infections in renal transplant recipients. Clin Microbiol Infect. (2016) 22:462.e5-9. doi: 10.1016/j.cmi.2016. 01.014

13. Kicia M, Wesolowska M, Kopacz Z, Kváč M, Sak B, Sokulska M, et al. Disseminated infection of encephalitozoon cuniculi associated with

\section{FUNDING}

This work was supported by the Ministry of Health subvention (grant numbers STM.A060.20.093 and STM.A060.20.105) from the IT Simple system of Wroclaw Medical University and the Czech Science Foundation (GACR 20-10706S). The funders had no role in study design, data collection and analysis, decision to publish, or preparation of the manuscript.

\section{ACKNOWLEDGMENTS}

We would like to thank Robin and Barbara Royle for their help in the language correction of the text.

osteolysis of hip periprosthetic tissue. Clin Infect Dis. (2018) 67:122834. doi: 10.1093/cid/ciy256

14. Levine A, Koletzko S, Turner D, Escher JC, Cucchiara S, de Ridder L, et al. The ESPGHAN revised porto criteria for the diagnosis of inflammatory bowel disease in children and adolescents. J Pediatr Gastroenterol Nutr. (2013) 58:795-806. doi: 10.1097/MPG.0000000000000239

15. Didier ES, Orenstein JM, Aldras A, Bertucci D, Rogers LB, Janney FA. Comparison of three staining methods for detecting microsporidia in fluids. $J$ Clin Microbiol. (1995) 33:3138-45. doi: 10.1128/jcm.33.12.3138-3145.1995

16. Didier ES, Vossbrinck CR, Baker MD, Rogers LB, Bertucci DC, Shadduck JA. Identification and characterization of three encephalitozoon cuniculi strains. Parasitology. (1995) 111:411-21. doi: 10.1017/S0031182000065914

17. Katzwinkel-Wladarsch S, Lieb M, Helse W, Löscher T, Rinder H. Direct amplification and species determination of microsporidian DNA from stool specimens. Trop Med Int Health. (1996) 1:373-8. doi: 10.1046/j.1365-3156.1996.d01-51.x

18. Buckholt MA, Lee JH, Tzipori S. Prevalence of enterocytozoon bieneusi in swine: an 18-month survey at a slaughterhouse in massachusetts. Appl Environ Microbiol. (2002) 68:2595-9. doi: 10.1128/AEM.68.5.2595-2599.2002

19. Hasani Z, Aghdaei HA, Balaii H, Azimirad M, Mirsamadi ES, Mirjalali H, et al. The first study on opportunistic intestinal microsporidiosis in IBD patients receiving immunosuppressive medications in Iran. Epidemiol Infect. (2017) 145:2095-9. doi: 10.1017/S0950268817000954

20. Zeissig S, Bürgel N, Günzel D, Richter J, Mankertz J, Wahnschaffe U, et al. Changes in expression and distribution of claudin 2, 5 and 8 lead to discontinuous tight junctions and barrier dysfunction in active Crohn's disease. Gut. (2007) 56:61-72. doi: 10.1136/gut.2006.094375

21. Furr AE, Ranganathan S, Finn OJ. Aberrant expression of MUC1 mucin in pediatric inflammatory bowel disease. Pediatr Dev Pathol. (2010) 13:2431. doi: 10.2350/08-06-0479.1

22. Valencakova A, Halanova M. Immune response to encephalitozoon infection review. Comp Immunol Microbiol Infect Dis. (2012) 35:17. doi: 10.1016/j.cimid.2011.11.004

23. Brdíčková K, Sak B, Holubová N, Květonová D, Hlásková L, Kicia M, et al. Encephalitozoon cuniculi genotype II concentrates in inflammation foci. $J$ Inflamm Res. (2020) 13:583-93. doi: 10.2147/JIR.S271628

24. Ferreira J, Baden R, Magge S, Cheifetz A. Cryptosporidiosis masquerading as a Crohn's flare. Inflamm Bowel Dis. (2011) 17:133-4. doi: 10.1002/ibd.21811

25. Sak B, Brady D, Pelikánová $M$, Květonová $D$, Rost $M$, Kostka $M$, et al. Unapparent microsporidial infection among immunocompetent humans in the Czech Republic. J Clin Microbiol. (2011) 49:106470. doi: 10.1128/JCM.01147-10

26. Matos O, Lobo ML, Xiao L. Epidemiology of enterocytozoon bieneusi infection in humans. J Parasitol Res. (2012) 2012:981424. doi: 10.1155/2012/981424

27. He Q, Leitch GJ, Visvesvara GS, Wallace S. Effects of nifedipine, metronidazole, and nitric oxide donors on spore germination and cell culture infection of the microsporidia encephalitozoon hellem and 
encephalitozoon intestinalis. Antimicrob Agents Chemother. (1996) 40:17985. doi: 10.1128/AAC.40.1.179

28. Canning EU, Hollister WS. Enterocytozoon bieneusi (Microspora): prevalence and pathogenicity in AIDS patients. Trans $R$ Soc Trop Med Hyg. (1990) 84:181-6. doi: 10.1016/0035-9203(90)90247-C

29. Lallo MA, Vidoto da Costa LF, Manoel de Castro J. Effect of three drugs against encephalitozoon cuniculi infection in immunosuppressed mice. Antimicrob Agents Chemother. (2013) 57:3067-71. doi: 10.1128/AAC.00 157-13

30. Costa SF, Weiss LM. Drug treatment of microsporidiosis. Drug Resist Updat. (2000) 3:384-99. doi: 10.1054/drup.2000.0174

31. Molina J-M., Tourneur M, Sarfati C, Chevret S, de Gouvello A, et al. Fumagillin treatment of intestinal microsporidiosis. N Engl J Med. (2002) 346:1963-9. doi: 10.1056/NEJMoa012924

32. Kicia M, Wesolowska M, Jakuszko K, Kopacz Z, Sak B, Květonová D, et al. Concurrent infection of the urinary tract with encephalitozoon cuniculi and enterocytozoon bieneusi in a renal transplant recipient. J Clin Microbiol. (2014) 52:1780-2. doi: 10.1128/JCM.03328-13
Conflict of Interest: The authors declare that the research was conducted in the absence of any commercial or financial relationships that could be construed as a potential conflict of interest.

Publisher's Note: All claims expressed in this article are solely those of the authors and do not necessarily represent those of their affiliated organizations, or those of the publisher, the editors and the reviewers. Any product that may be evaluated in this article, or claim that may be made by its manufacturer, is not guaranteed or endorsed by the publisher.

Copyright (c) 2021 Zajaczkowska, Akutko, Kváč, Sak, Szydłowicz, Hendrich, Iwańczak and Kicia. This is an open-access article distributed under the terms of the Creative Commons Attribution License (CC BY). The use, distribution or reproduction in other forums is permitted, provided the original author(s) and the copyright owner(s) are credited and that the original publication in this journal is cited, in accordance with accepted academic practice. No use, distribution or reproduction is permitted which does not comply with these terms. 